\title{
Optical Thomas-Reiche-Kuhn Sum Rules
}

\author{
Stephen M. Barnett \\ Department of Physics, Unniversity of Strathclyde, Glasgow G4 ONG, United Kingdom
}

Rodney Loudon

School of Computer Science and Electronic Engineering, University of Essex, Colchester CO4 3SQ, United Kingdom

(Received 12 September 2011; published 6 January 2012)

\begin{abstract}
The Thomas-Reiche-Kuhn sum rule is a fundamental consequence of the position-momentum commutation relation for an atomic electron and it provides an important constraint on the transition matrix elements for an atom. Analogously, the commutation relations for the electromagnetic field operators in a magnetodielectric medium constrain the properties of the dispersion relations for the medium through four sum rules for the allowed phase and group velocities for polaritons propagating through the medium. These rules apply to all bulk media including the metamaterials designed to provide negative refractive indices. An immediate consequence of this is that it is not possible to construct a medium in which all the polariton modes for a given wavelength lie in the negative-index region.
\end{abstract}

DOI: 10.1103/PhysRevLett.108.013601

PACS numbers: $42.50 . \mathrm{Ct}, 71.36 .+\mathrm{c}$, 78.67.Pt

Sum rules and their continuum, integral analogues provide a simple and reliable method with which to check the validity of model calculations and to ensure that important physical principles are respected. The famous KramersKronig relations [1], for example, enforce causality and provide a relationship between the real and imaginary parts of linear response functions. The fluctuation-dissipation theorem, for another, tells us the lowest amount of noise required to accompany losses so that quantum mechanical commutation relations are conserved [2]. Radiative processes in atoms and molecules are also constrained by sum rules. The extent to which spontaneous emission rates can be modified by environmental influences is constrained so that suppression of decay at some frequencies is always balanced by enhanced decay at other frequencies [3].

The Thomas-Reiche-Kuhn sum rule [4,5] and its relatives [6-9] play an especially important role in the interaction between light and matter. In its simplest form it states that the sum of the squares of the dipole matrix moments from any energy level, weighted by the corresponding energy differences, is a constant. The sum rule is a fundamental consequence, for an atomic electron, of the canonical commutation relation between the position and momentum. Indeed, we can also view it as a condition that is necessary in order for this commutation relation to hold. Among the many consequences of this sum rule we note that it constrains the cross sections for absorption and stimulated emission [5].

The quantum nature of the electromagnetic field manifests itself in the commutation relations for the field operators. In particular, in a dielectric medium, the $\mathbf{E}, \mathbf{D}$, and $\mathbf{H}$ fields satisfy the equal-time commutation relations $[10,11]$

$$
\begin{aligned}
{\left[\mu_{0} H_{j}(\mathbf{r}, t), \varepsilon_{0} E_{k}\left(\mathbf{r}^{\prime}, t\right)\right] } & =i \hbar \varepsilon_{j k l} \nabla_{l} \delta\left(\mathbf{r}-\mathbf{r}^{\prime}\right), \\
{\left[\mu_{0} H_{j}(\mathbf{r}, t), D_{k}\left(\mathbf{r}^{\prime}, t\right)\right] } & =i \hbar \varepsilon_{j k l} \nabla_{l} \delta\left(\mathbf{r}-\mathbf{r}^{\prime}\right),
\end{aligned}
$$

where $\varepsilon_{j k l}$ is the permuting symbol and we have adopted the familiar summation convention [12]. The excitations of the field in the medium, the polaritons, are coupled modes of the electromagnetic field and the polarization of the medium [13-15]. For simplicity, we specialize to homogenous and isotropic media so that the polariton properties are the same for all propagation directions. A generalization to anisotropic media would introduce direction-dependent phase and group velocities. The dispersion relation provides, in general, multiple frequencies $\omega_{j}$ for each wave number $k$, one for each polariton mode. A magnetic medium is described in terms of a magnetic induction $\mathbf{B}$ in addition to the magnetic field $\mathbf{H}$. This adds to the relations (1) the further and distinct commutation relations

$$
\begin{aligned}
{\left[B_{j}(\mathbf{r}, t), \varepsilon_{0} E_{k}\left(\mathbf{r}^{\prime}, t\right)\right] } & =i \hbar \varepsilon_{j k l} \nabla_{l} \delta\left(\mathbf{r}-\mathbf{r}^{\prime}\right), \\
{\left[B_{j}(\mathbf{r}, t), D_{k}\left(\mathbf{r}^{\prime}, t\right)\right] } & =i \hbar \varepsilon_{j k l} \nabla_{l} \delta\left(\mathbf{r}-\mathbf{r}^{\prime}\right) .
\end{aligned}
$$

The magnetic effects add, to the polaritons, further coupled modes for the electromagnetic field and the material medium. For simplicity, we follow precedent and refer to all of these modes collectively as polaritons [15].

Milonni and Maclay have provided explicit expressions for the four electric and magnetic field operators in a lossless magnetodielectric medium [16,17]:

$$
\begin{aligned}
& \mathbf{E}(\mathbf{r}, t)=i \sum_{\mathbf{k}, \lambda, j} \omega_{j} A_{\mathbf{k}, \lambda, j} e^{-i\left(\omega_{j} t-\mathbf{k} \cdot \mathbf{r}\right)} \mathbf{e}_{\mathbf{k}, \lambda}+\text { H.c., } \\
& \mathbf{D}(\mathbf{r}, t)=i \sum_{\mathbf{k}, \lambda, j} \varepsilon_{0} \varepsilon\left(\omega_{j}\right) \omega_{j} A_{\mathbf{k}, \lambda, j} e^{-i\left(\omega_{j} t-\mathbf{k} \cdot \mathbf{r}\right)} \mathbf{e}_{\mathbf{k}, \lambda}+\text { H.c., } \\
& \mathbf{H}(\mathbf{r}, t)=i \sum_{\mathbf{k}, \lambda, j} \frac{A_{\mathbf{k}, \lambda, j}}{\mu_{0} \mu\left(\omega_{j}\right)} e^{-i\left(\omega_{j} t-\mathbf{k} \cdot \mathbf{r}\right)} \mathbf{k} \times \mathbf{e}_{\mathbf{k}, \lambda}+\text { H.c. } \\
& \mathbf{B}(\mathbf{r}, t)=i \sum_{\mathbf{k}, \lambda, j} A_{\mathbf{k}, \lambda, j} e^{-i\left(\omega_{j} t-\mathbf{k} \cdot \mathbf{r}\right)} \mathbf{k} \times \mathbf{e}_{\mathbf{k}, \lambda}+\text { H.c., }
\end{aligned}
$$


where the summation runs over all the allowed wave vectors $(\mathbf{k})$, polarizations $(\lambda)$, and polariton branches $(j)$. Here we have introduced the operators

$$
A_{\mathbf{k}, \lambda, j}=\left(\frac{\hbar \mu_{0} \mu}{2 V} \frac{1}{k} \frac{d \omega_{j}}{d k}\right)^{1 / 2} a_{\mathbf{k}, \lambda, j},
$$

where $V$ is the quantization volume and the frequencies for each of the polaritons are the solutions of the dispersion relation

$$
\omega^{2} \varepsilon(\omega) \mu(\omega)-k^{2} c^{2}=0,
$$

one for each polariton branch. If we insert these expressions into our four fundamental commutation relations (1) and (2) and take the limit of infinite volume in the standard manner [18] then, in analogy with the atomic ThomasReiche-Kuhn sum rules, we find the correct commutation relations only if we impose the four polariton sum rules:

$$
\frac{1}{c^{2}} \sum_{j} z\left(\omega_{j}\right) \frac{\omega_{j}}{k} \frac{d \omega_{j}}{d k}=1,
$$

where $z\left(\omega_{j}\right)$ represents any of the four functions $1, \varepsilon\left(\omega_{j}\right)$, $\mu\left(\omega_{j}\right)$, and $\varepsilon\left(\omega_{j}\right) \mu\left(\omega_{j}\right)$, and the summation runs over all the polariton modes. The first and last of these have been found previously for nonmagnetic media [19-21] and can be expressed equivalently in terms of the allowed polariton phase and group velocities:

$$
\begin{aligned}
& \sum_{j} \frac{\omega_{j}}{k} \frac{d \omega_{j}}{d k}=\sum_{j} v_{g}^{j} v_{p}^{j}=c^{2} \\
& \sum_{j} \frac{k}{\omega_{j}} \frac{d \omega_{j}}{d k}=\sum_{j} \frac{v_{g}^{j}}{v_{p}^{j}}=1 .
\end{aligned}
$$

Our task is to prove the four sum rules (6) for magnetodielectric media. In doing so, it is advantageous to prove a generalized result that applies to more realistic media exhibiting losses caused by absorption. For such media, the relative permittivity, $\varepsilon(\omega)$ and permeability $\mu(\omega)$ are complex, as is the frequency $\omega$ associated with each polariton mode. The sum rules (6) then apply to the real parts of the summands.

To provide a proof of the four sum rules (6) we require the use of two properties of the relative permittivity and permeability as functions of the complex frequency $\omega$. The first of these properties is the familiar crossing relation [1],

$$
\varepsilon(-\omega)=\varepsilon^{*}\left(\omega^{*}\right), \quad \mu(-\omega)=\mu^{*}\left(\omega^{*}\right),
$$

which enforces the reality of the displacement and the magnetic induction. The second property, which is intimately connected with causality, is that both $\varepsilon(\omega)$ and $\mu(\omega)$ tend to unity as $|\omega| \rightarrow \infty$ and are analytic in the upper half of the complex $\omega$ plane. We restrict our analysis to situations in which they have only simple poles in the lower half-plane, each of which corresponds to a polariton mode [22].
Consider an integral of the form

$$
I_{z}=-\frac{1}{\pi i} \int_{-\infty}^{\infty} d \omega \frac{\omega z(\omega)}{\omega^{2} \varepsilon(\omega) \mu(\omega)-k^{2} c^{2}},
$$

where the function $z(\omega)$ again represents any of the four possibilities, $1, \varepsilon(\omega), \mu(\omega)$, or $\varepsilon(\omega) \mu(\omega)$. It is natural to evaluate this integral by closing a semicircular contour either in the upper or lower half of the complex $\omega$ plane. The two integration contours are presented in Fig. 1. Naturally, the result must be the same whichever method is used. We start with the integral in the upper half-plane. The zeros of the denominator in the integrand all lie in the lower half of the complex $\omega$ plane. This, and the fact that $z(\omega)$ is also analytic in the upper half-plane, means that the integrand is analytic in the upper half-plane and hence that the integral around this contour must be zero. It then follows that

$$
\begin{aligned}
& -\frac{1}{\pi i} \int_{-R}^{R} d \omega \frac{\omega z(\omega)}{\omega^{2} \varepsilon(\omega) \mu(\omega)-k^{2} c^{2}} \\
& =\frac{1}{\pi i} \int_{0}^{\pi} i \operatorname{Re}^{i \theta} d \theta \frac{\operatorname{Re}^{i \theta} z\left(\operatorname{Re}^{i \theta}\right)}{R^{2} e^{i 2 \theta} \varepsilon\left(\operatorname{Re}^{i \theta}\right) \mu\left(\operatorname{Re}^{i \theta}\right)-k^{2} c^{2}} \Rightarrow I_{z} \\
& =1,
\end{aligned}
$$
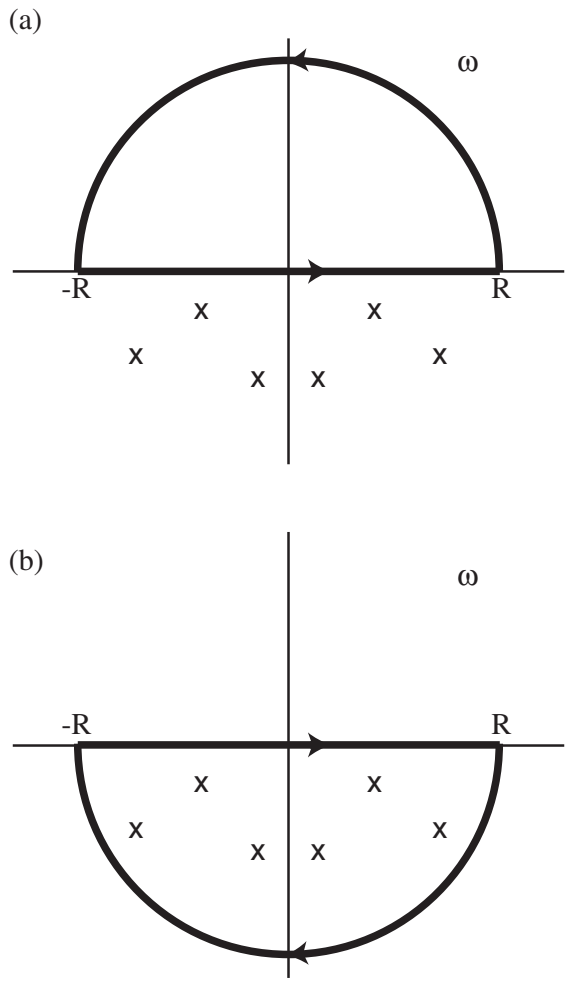

FIG. 1. The two integration contours used in proving our sum rules, (a) in the upper half-plane and (b) in the lower. Note that all the poles of the integrand, represented by the crosses, lie in the lower half of the complex $\omega$ plane and exist in pairs, $\omega_{j}$ and $-\omega_{j}^{*}$. 
where we have written $\omega=\operatorname{Re}^{i \theta}$ on the semicircular path and used the fact that $\varepsilon\left(\operatorname{Re}^{i \theta}\right), \mu\left(\operatorname{Re}^{i \theta}\right) \rightarrow 1$ as $R \rightarrow \infty$.

We can also evaluate $I_{z}$ by closing a semicircular contour in the lower half of the complex plane. Note that doing so will introduce residues from the zeros of the denominator, corresponding to the solutions of the dispersion relation (5). These will appear with weight $-2 \pi i$ with the minus sign arising because the sense of integration around the contour is clockwise [12]. We find

$$
\begin{aligned}
& -\frac{1}{\pi i} \int_{-R}^{R} d \omega \frac{\omega z(\omega)}{\omega^{2} \varepsilon(\omega) \mu(\omega)-k^{2} c^{2}} \\
& =-\frac{1}{\pi i} \int_{\pi}^{2 \pi} i \operatorname{Re}^{i \theta} d \theta \frac{\operatorname{Re}^{i \theta} z\left(\operatorname{Re}^{i \theta}\right)}{R^{2} e^{i 2 \theta} \varepsilon\left(\operatorname{Re}^{i \theta}\right) \mu\left(\operatorname{Re}^{i \theta}\right)-k^{2} c^{2}} \\
& \quad+2 \mathcal{R}_{z},
\end{aligned}
$$

where $\mathcal{R}_{z}$ is the sum of the residues of the integrand at the poles given by the dispersion relation (5). Note that the poles of $z(\omega)$ do not contribute to the residues as the functions $\varepsilon(\omega)$ and $\mu(\omega)$ are present in the denominator of the integrand so the integrand is regular at these points. Taking the limit as $R$ tends to infinity we are left with

$$
I_{z}=-1+2 \mathcal{R}_{z}
$$

It follows, therefore, that $\mathcal{R}_{z}=1$.

It only remains to calculate the required residues of

$$
\begin{aligned}
\frac{\omega z(\omega)}{\omega^{2} \varepsilon(\omega) \mu(\omega)-k^{2} c^{2}}= & \frac{z(\omega) \omega}{2 k c}\left(\frac{1}{\omega n(\omega)-k c}\right. \\
& \left.-\frac{1}{\omega n(\omega)+k c}\right),
\end{aligned}
$$

where $n(\omega)$ is the (complex) refractive index, $\sqrt{\varepsilon(\omega) \mu(\omega)}$. We can do this rather neatly by using a simple trick [12]: the residue of a function $1 / f(\omega)$ at a simple pole $\omega_{0}$ is $d f / d \omega$ evaluated at $\omega_{0}$. It follows that

$$
\begin{aligned}
\mathcal{R}_{z} & =\sum_{\text {poles }} \frac{\omega z(\omega)}{2 k c}\left(\left.\frac{1}{\frac{d \omega n(\omega)}{d \omega}}\right|_{\omega n=k c}-\left.\frac{1}{\frac{d \omega n(\omega)}{d \omega}}\right|_{\omega n=-k c}\right) \\
& =\frac{1}{2 c^{2}} \sum_{\text {poles }} z(\omega) \frac{\omega}{k} \frac{d \omega}{d k},
\end{aligned}
$$

where the sum runs over all the poles, at positions corresponding to the solutions of the dispersion relation (5). Finally, we note that the summation runs over pairs of complex frequencies $\omega_{j}$ and $-\omega_{j}^{*}$, with each pair corresponding to a single polariton branch. We can rewrite Eq. (14) as a sum over these pairs:

$$
\begin{aligned}
\mathcal{R}_{z} & =1=\frac{1}{2 c^{2}} \sum_{j}\left(z\left(\omega_{j}\right) \frac{\omega_{j}}{k} \frac{d \omega_{j}}{d k}+z\left(-\omega_{j}^{*}\right) \frac{-\omega_{j}^{*}}{k} \frac{d-\omega_{j}^{*}}{d k}\right) \\
& =\frac{1}{c^{2}} \operatorname{Re}\left(\sum_{j} z\left(\omega_{j}\right) \frac{\omega_{j}}{k} \frac{d \omega_{j}}{d k}\right),
\end{aligned}
$$

where we have used the crossing relation (8). Our four possible values of $z(\omega)$ give the four required sum rules, reducing to Eq. (6) for a lossless medium.

For the limiting case of a lossless medium, the simplest model of a magnetodielectric medium has a single electric and a single magnetic resonance, so that the relative permittivity and permeability are [15]

$$
\varepsilon(\omega)=\frac{\omega_{\mathrm{Le}}^{2}-\omega^{2}}{\omega_{\mathrm{Te}}^{2}-\omega^{2}}, \quad \mu(\omega)=\frac{\omega_{\mathrm{Lm}}^{2}-\omega^{2}}{\omega_{\mathrm{Tm}}^{2}-\omega^{2}},
$$

where the $\mathrm{L}$ and $\mathrm{T}$ subscripts denote the longitudinal and transverse frequencies associated with the electric and magnetic dipole resonances e and $m$, respectively. The associated dispersion relation, given by Eq. (5), has three (positive) solutions, $\omega_{1}, \omega_{2}$, and $\omega_{3}$ for each value of $k$, associated with three distinct polariton branches. The dispersion relation for this relative permittivity and permeability is a cubic equation in $\omega^{2}$ with

$$
\sum_{j} \omega_{j}^{2}=\omega_{\mathrm{Le}}^{2}+\omega_{\mathrm{Lm}}^{2}+k^{2} c^{2} .
$$

Differentiating this equation with respect to $k$ gives, directly, the first of our four sum rules. The product of our three roots also follows directly from the dispersion relation:

$$
\prod_{j} \omega_{j}^{2}=k^{2} c^{2} \omega_{\mathrm{Te}}^{2} \omega_{\mathrm{Tm}}^{2}
$$

Taking the logarithm of this expression and then differentiating with respect to $k$ gives the last of our four sum rules. Our second and third sum rules are of similar form and so we need only calculate one of them in order to verify the accuracy of them both. The second sum rule, for our model medium, is

$$
\begin{aligned}
\sum_{j} \varepsilon\left(\omega_{j}\right) \frac{\omega_{j}}{k} \frac{d \omega_{j}}{d k} & =c^{2}+\sum_{j}\left(\frac{\omega_{\mathrm{Te}}^{2}-\omega_{\mathrm{Le}}^{2}}{\omega_{\mathrm{Te}}^{2}-\omega_{j}^{2}}\right) \frac{\omega_{j}}{k} \frac{d \omega_{j}}{d k} \\
& =c^{2}-\frac{\omega_{\mathrm{Te}}^{2}-\omega_{\mathrm{Le}}^{2}}{2 k} \frac{d}{d k} \ln \prod_{j}\left(\omega_{\mathrm{Te}}^{2}-\omega_{j}^{2}\right) \\
& =c^{2}
\end{aligned}
$$

because the product of roots in the logarithm is independent of $k$.

We note that our sum-rules apply to the newly developed metamaterials including those designed to realize negative refractive indices $[17,23,24]$, provided only that the wavelength is large enough to allow a description in terms of $\varepsilon(\omega)$ and $\mu(\omega)$. For a negative refractive index to occur we require both $\varepsilon(\omega)$ and $\mu(\omega)$ to be negative. Our example from the preceding paragraph, therefore, suffices to demonstrate this behavior. If, in the relative permittivity and permeability (16), there are frequencies lying between $\omega_{\text {Le }}$ and $\omega_{\mathrm{Te}}$ and also between $\omega_{\mathrm{Lm}}$ and $\omega_{\mathrm{Tm}}$ then polaritons at these frequencies will experience a negative refractive 


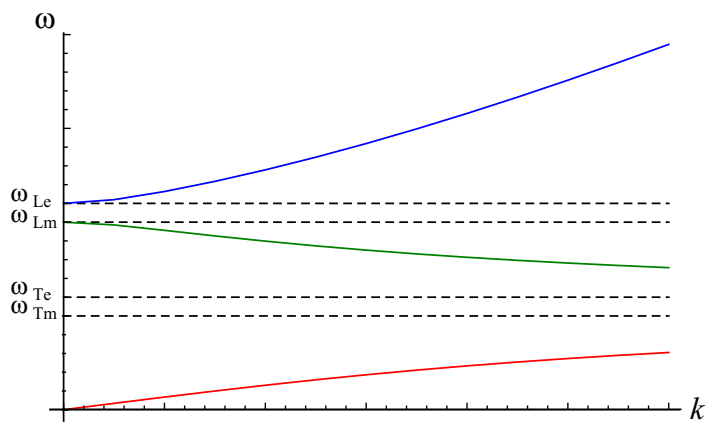

FIG. 2 (color online). Polariton dispersion curves for a lossless medium, where the permittivity and permeability are given by Eq. (16). The relative magnitudes of the four constants have been chosen so as to give a negative-index frequency range between $\omega_{\mathrm{Te}}$ and $\omega_{\mathrm{Lm}}$. Note that in this negative-index region the polariton branch has a negative slope.

index. The three polariton branches in such a case are presented in Fig. 2. We see that there is a polariton in the negative-index region for all values of $k$ and that $\frac{d \omega}{d k}$ is negative for this branch [25]. It follows that the corresponding terms in our first and fourth sum rules will be negative and must be compensated by correspondingly larger positive terms for polaritons outside this region. This tells us, also, that we cannot construct a medium in which all the polariton modes for a given wavelength lie in the negative-index region. All of the terms in our second and third sum rules will be positive, however, as $\varepsilon(\omega)$ and $\mu(\omega)$ are negative for the negative-index polariton.

Our derivation has provided not only the four sum rules, Eq. (6), for lossless media, but has also indicated the required modified forms, Eq. (15), when losses are important. A recent analysis of the quantized electromagnetic field for a lossy magnetodielectric medium [26] has provided explicit forms for the electric, magnetic, displacement, and magnetic induction fields. In a future publication, we shall show how our sum rules, Eq. (15), are necessary to arrive at the required field commutation relations in lossy media. Alternatively, more in the spirit of the Thomas-Reiche-Kuhn sum rules, we can enforce the commutation relations for the Kheirandish-Soltani model and thereby provide an alternative derivation of our sum rules.

We thank Alison Yao and Peter Milonni for helpful comments and suggestions. We also express our gratitude to an anonymous referee who kindly checked our calculations and pointed out a number of errors in our original manuscript. This work was supported by the U.K. Engineering and Physical Sciences Research Council, the Royal Society, and the Wolfson Foundation.
[1] J.D. Jackson, Classical Electrodynamics (Wiley, New York, 1999), 3rd ed.

[2] L. Landau and E. M. Lifshitz, Statistical Physics (Butterworth Heinemann, Oxford, 1980), 3rd ed., Pt. 1.

[3] S. M. Barnett and R. Loudon, Phys. Rev. Lett. 77, 2444 (1996); Quantum Semiclass. Opt. 10, 591 (1998).

[4] E. U. Condon and G. H. Shortley, The Theory of Atomic Spectra (Cambridge University Press, Cambridge, 1953), p. 108.

[5] E. Merzbacher, Quantum Mechanics (Wiley, New York, 1970), 2nd ed., p. 457.

[6] H. A. Bethe and E. E. Salpeter, Quantum Mechanics of One- and Two-Electron Atoms (Dover, New York, 2008), p. 256.

[7] A. M. Stoneham, Theory of Defects in Solids (Oxford University Press, Oxford, 2001), p. 884.

[8] G. Orlandini and M. Traini, Rep. Prog. Phys. 54, 257 (1991).

[9] C. Baxter, J. Phys. B 25, L589 (1992).

[10] W. Heisenberg, The Physical Principles of the Quantum Theory (Dover, New York, 1949), p. 183.

[11] D.P. Craig and T. Thirunamachandran, Molecular Quantum Electrodynamics (Dover, New York, 1998), p. 40.

[12] G. Stephenson and P. M. Radmore, Advanced Mathematical Methods for Engineering and Science Students (Cambridge University Press, Cambridge, 1990).

[13] J. J. Hopfield, Phys. Rev. 112, 1555 (1958).

[14] C. Kittel, Quantum Theory of Solids (Wiley, New York, 1987), 2nd ed., p. 44.

[15] D. L. Mills and E. Burstein, Rep. Prog. Phys. 37, 817 (1974).

[16] P. W. Milonni, J. Mod. Opt. 42, 1991 (1995).

[17] P. W. Milonni and G. J. Maclay, Opt. Commun. 228, 161 (2003).

[18] R. Loudon, The Quantum Theory of Light (Oxford University Press, Oxford, 2000), 3rd ed., p. 144.

[19] B. Huttner, J. J. Baumberg, and S. M. Barnett, Europhys. Lett. 16, 177 (1991).

[20] B. Huttner and S. M. Barnett, Phys. Rev. A 46, 4306 (1992).

[21] O. Al-Dossary, M. Babiker, and N. Enfati, Phys. Rev. A 54, 2419 (1996).

[22] Poles of second or higher order, should they exist, may be dealt with by separating them into the corresponding number of nearby simple poles and then applying to the sum rules the limit as they converge to the same point in the complex $\omega$ plane.

[23] V. G. Veselago, Sov. Phys. Usp. 10, 509 (1968).

[24] P. W. Milonni, Fast Light, Slow Light and Left-Handed Light (Taylor and Francis, New York, 2005).

[25] The phase velocity is negative and the group velocity is positive in a negative-index medium [24]. Here, therefore, it is the polariton propagating in the opposite sense to those outside the negative-index region that contributes to the sum rule.

[26] F. Kheirandish and M. Soltani, Phys. Rev. A 78, 012102 (2008). 